\title{
eJRIEPS
}

Ejournal de la recherche sur l'intervention en éducation physique et sport

$23 \mid 2011$

Varia

\section{Approche technologique des techniques individuelles et collectives en Football. L'exemple du jeu en déviation}

\section{Alain Lemoine}

\section{(2) OpenEdition}

\section{Édition électronique}

URL : http://journals.openedition.org/ejrieps/4561

DOI : $10.4000 /$ ejrieps.4561

ISSN : 2105-0821

\section{Éditeur}

ELLIADD

\section{Référence électronique}

Alain Lemoine, «Approche technologique des techniques individuelles et collectives en Football. L'exemple du jeu en déviation », eJRIEPS [En ligne], 23 | 2011, mis en ligne le 01 avril 2011, consulté le 21 février 2020. URL : http://journals.openedition.org/ejrieps/4561 ; DOI : 10.4000/ejrieps.4561

La revue eJRIEPS est mise à disposition selon les termes de la Creative Commons Attribution 4.0 International License. 
Approche technologique des techniques individuelles et collectives en Football

\section{L'exemple du jeu en déviation}

Alain Lemoine

Laboratoire de Recherches " EA 3300 : APS et Conduites Motrices : Adaptations et Réadaptations ", Université de Picardie Jules Verne, Faculté des Sciences du sport, 80025 Amiens, France

\section{Résumé}

Nous présenterons ici nos travaux concernant l'observation et l'analyse du jeu en déviation où la technique individuelle du joueur devient une maitrise collective “ supérieure » qui permet à l'attaque de prendre un avantage spatio-temporel sur la défense. Cette recherche a une dimension technologique dans la mesure où elle aborde plusieurs registres dont principalement celui de maitrise. Partant du réel, elle a permis d'identifier des types d'attaques et d'en donner les principes. Nous voyons ici tout l'intérêt que cela présente pour l'intervenant en STAPS ou les enseignants qui pourront ainsi proposer aux élèves des contenus nécessaires à la construction des compétences telles qu'elles sont définies par les programmes de la discipline.

\section{Introduction}

L'intervenant en Sciences et Techniques des Activités Physiques et Sportives (Staps), l'enseignant, l'entraîneur peuvent aujourd'hui puiser dans une multitude d'approches théoriques pour expliquer, analyser et enseigner les sports collectifs. Parlebas (1967) propose la notion de socio-motricité où « l'interaction motrice avec partenaire (s) et/ou adversaire (s) est nécessaire dans l'accomplissement de la tâche ». Teodoresco (1977) a systématisé le déroulement des oppositions de deux équipes en identifiant des phases et des principes. Deleplace (1979) dont les recherches s'apparente à une démarche technologique a ouvert la voie à des travaux féconds menés par Bouthier (1989) qui fait la liaison avec l'intervention des enseignants en proposant une Pédagogie des Modèles de Décisions Tactiques. Dans la même logique de relation avec la didactique des acticités physiques et sportives Eloi \& Uhlrich proposent une définition des sports collectifs s'appuyant sur la notion d'essence de l'activité où c'est «la simultanéité des actions engagées par les deux équipes qui organise le véritable enjeu des sports collectifs » (Eloi \& Uhlrich, 2001, 117). II en découle des principes liés à la marque, à la cible, à l'espace de 


\section{eJRIEPS 23 avril 2011}

jeu utilisables par l'enseignant. Gréhaigne, quant à lui, interroge les théories dynamiques qui expliquent que l'élève doit comprendre «que c'est la mouvance de tous les joueurs qu'il faut saisir, la forme purement instantanée de la configuration et non pas la réduire à une figure statique hors de son contexte » $(2009,15)$.

Les approches théoriques ne sont pas les seuls modèles sur lesquels l'enseignant peut s'appuyer dans son travail didactique de transmission des savoirs aux élèves et il peut aussi prendre en compte les modèles issus de la pratique comme le fait Eloi (2009) quand il s'appuie sur la définition du genre et du style du passeur en volley-ball afin de replacer «la technique spécifique » (Eloi, 2009, 103) dans une logique stratégico-tactique du joueur.

Mais quelle est la place faite à la technique dans ce contexte ? A partir de quoi, l'enseignant qui doit amener les élèves à construire des compétences motrices combinant des connaissances, des attitudes et des capacités telles qu'elles sont décrites par les programmes d'enseignement de l'EPS peut il déterminer ce qu'il doit transmettre?

Son interrogation portera alors sur la nature des savoirs mobilisés et l'identification des techniques comme « des manières de faire, des procédés visant l'efficacité ; un ensemble de repères stables permettant répétition et affinement de l'action » (Bouthier \& Durey, 1994, 111). Si la technique sportive transmise et enseignée fait le plus souvent référence à une efficience corporelle fruit d'habitus culturels et d'avancées technologiques reconnues pour leur efficacité sur le terrain, elle est plus que cela et l'unicité du mot cache une pluralité des objets (Combarnous, 1982). On peut avancer alors la notion de technicité qui se décompose en quatre registres : la participation, la lecture, la transformation et la maîtrise (Martinand, 1993). Ce dernier registre servira de base à notre réflexion dans la mesure où les joueurs de football utilisent la passe en déviation qui est une techniques corporelles en usage, habileté définie et répertoriée par les experts, décrites dans les ouvrages et faisant l'objet d'un apprentissage.

Nous explorerons le football et plus particulièrement l'observation, l'analyse et la compréhension des mouvements collectifs de 3 à 4 joueurs quand ils sont impliqués dans des séquences de jeu à une touche de balle débouchant sur un tir au but.

En nous référant aux travaux de Russo (1986) quant à l'élaboration de travaux technologiques, nous élaborerons un outil d'observation des faits de terrain nécessaire à une analyse méthodiques de séquences vidéo sélectionnées. Puis nous ferons émerger un corpus de connaissances composé de la classification des attaques et des principes tactiques identifiés comme étant nécessaires à la réalisation des attaques en déviation. 


\section{eJRIEPS 23 avril 2011}

\section{Méthode}

1. 1 Elaboration d'un outil d'observation

C'est un préambule nécessaire à la transmission des connaissances et à la communication entre intervenants. Dans notre cas, il s'agit de repérer la direction, le moment et les receveurs potentiels de chaque déviation. La grille de recueil de données est soumise aux protocoles scientifiques de validation (Falissard, 2001) qui montrent la reproductibilité intra-juge grâce à l'analyse de variance (0.5) et la concordance de jugements de différents experts mesurée par le test de Kappa ( $k=0.653)$. Huit types de déviations sont identifiées : un dans l'axe profond du terrain ; deux qui vont vers l'avant et les côtés ; deux qui écartent le ballon à $90^{\circ}$ vers les côtés du terrain et trois qui sont jouées en retrait par rapport à l'avancée de l'attaque.

\subsection{Observation méthodique des séquences vidéo}

Le visionnage de 40 attaques a permis d'identifier des configurations de jeu à des instants de l'attaque qui représentent un « micro-état du système attaque/défense faisant référence à la distribution des joueurs et du ballon sur le champ de jeu en fonction de leurs positions, leurs orientations et leurs vitesses de déplacement » (Gréhaigne \& Bouthier, 1994, 45). Ainsi repérés : la position des joueurs, le moment et la direction de la déviation renseignent sur les intentions tactiques ${ }^{1}$ des joueurs (fig.2). La représentation graphique des axes défensifs et offensifs, permet de visualiser à la fois la dimension synchronique des attaques (à un instant donné) et l'aspect diachronique (à plusieurs instants successifs) de son déroulement. La technique de déviation choisie par le joueur prend un sens car la passe est replacée dans le déroulement chronologique de l'attaque et nous pouvons alors expliquer les choix tactiques effectués par le porteur de balle quand il dévie la balle dans un espace particulier du terrain en rapport avec les positions respectives des attaquants et des défenseurs.

\footnotetext{
${ }^{1}$ C'est : « la méthode d'action propre au sujet en situation de jeu où pour remplir son rôle, le joueur s'efforce de subir au minimum les contraintes, les incertitudes et les hasards du jeu et d'utiliser au maximum les capacités, potentiels et compétences dont il dispose » Gréhaigne (2009, 142).
} 


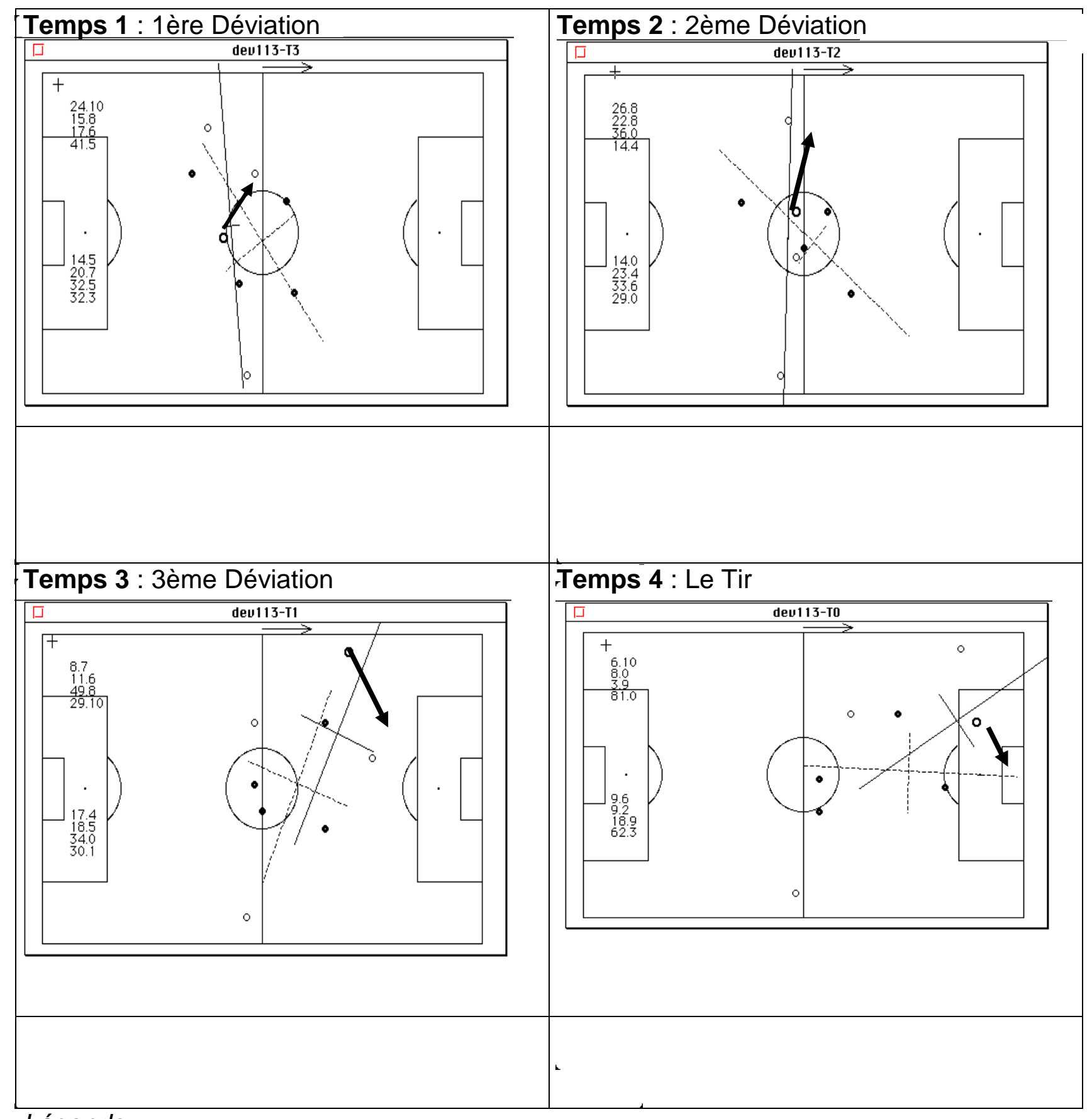

Légende:

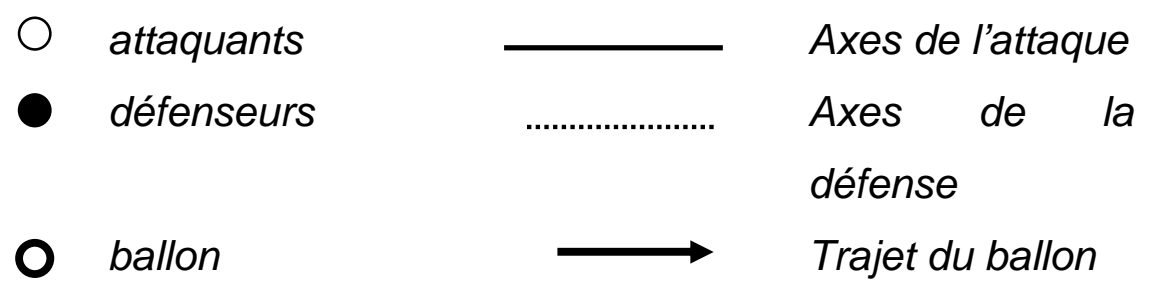

Figure 2. Relevé topographique final d'une attaque utilisant la technique de la déviation 


\section{eJRIEPS 23 avril 2011}

\section{Résultats}

\subsection{Les techniques utilisées}

Les résultats montrent que les déviations dans l'axe et la profondeur du terrain ne sont pas majoritairement utilisées (12,5\%). De manière privilégiée, ce sont les passes effectuées sur les côtés que les joueurs emploient pour aller vers la cible $(58,33 \%)$. Le jeu en retrait ne représente que 12,5\%, ce qui montre la primauté du jeu vers l'avant dans ce type d'attaque. Quant aux déviations latérales $(16,67 \%)$, si elles permettent l'évitement d'un adversaire, elles ralentissent la progression vers la cible en amenant le ballon vers les lignes de touche.

2. 2. La stabilité diachronique

$67,5 \%$ des attaques utilisant la technique de déviation n'ont pas ou très peu de modification de leurs EJE. Les joueurs occupent des positions relatives stables les uns par rapport aux autres du début à la fin de l'attaque.

2.3 Les différentes combinaisons

Le résultat du T de Student appliqué à la quantité d'incertitude produite par la succession des déviations utilisées dans les 40 combinaisons étudiées permet d’identifier trois types d'attaque (Fig. 3).

\section{Le corpus des connaissances}

3. 1. La classification des attaques (Fig. 3, 4 et 5)

Cette classification des attaques utilisant la technique de la déviation fait référence au registre de maitrise et elle permettra de contribuer «à la construction de compétences professionnelles » (Ulrich \& Bouthier, 2008, 202). 


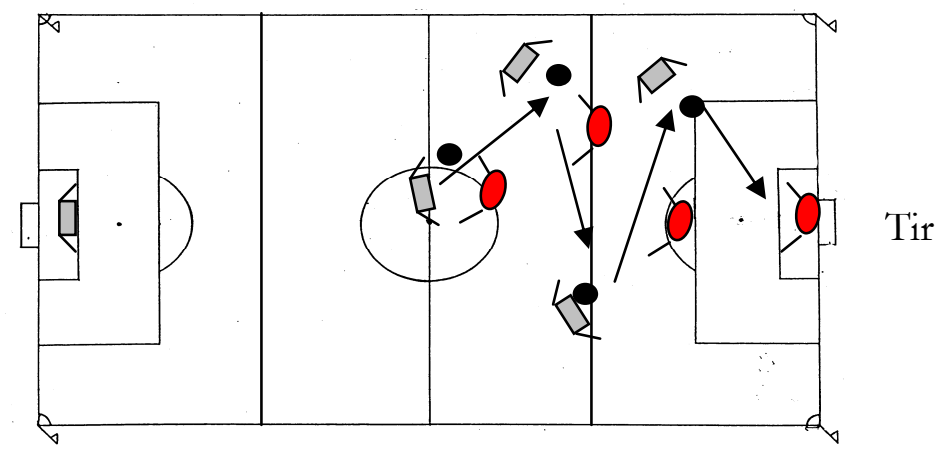

Figure 3 : Classification des attaques utilisant la technique de la déviation. Attaque de type I. Légende: La balle circule systématiquement vers l'avant et dans une direction opposée ( \pm 909 à la précédente. Les attaquants forment un Espace de Jeu Effectif ${ }^{2}$ (EJE) organisé sur la largeur du terrain en englobant l'EJE des défenseurs.

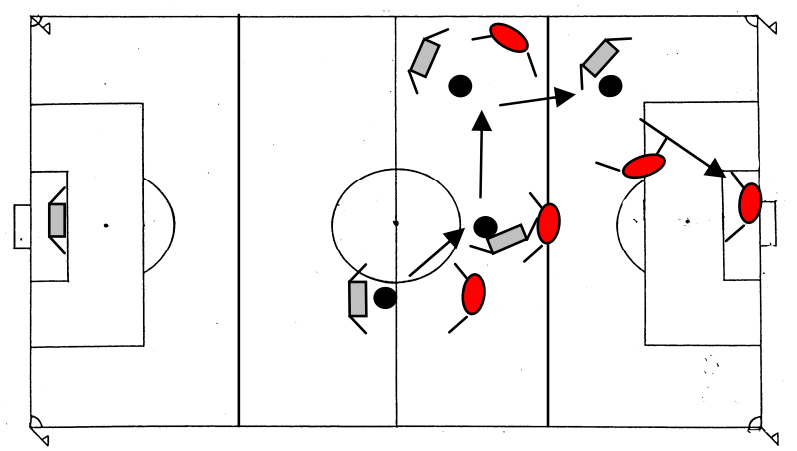

Figure 4 : Classification des attaques utilisant la technique de la déviation. Attaque de type II. Légende : La balle est déviée dans une direction systématiquement différente de la précédente, mais des déviations latérales écartent momentanément le ballon de l'accès le plus direct à la cible.



Figure 5. Classification des attaques utilisant la technique de la déviation. Attaque de type III. Légende: La balle est déviée au moins une fois à l'inverse du déroulement de l'attaque. Cela correspond à des attaques où la défense est très dense et oblige l'attaque à jouer en soutien pour conserver le ballon et progresser ensuite vers l'avant.

\footnotetext{
${ }^{2}$ Espace de Jeu Effectif (EJE) : il représente une surface polygonale délimitée par la position des joueurs d'une même équipe à l'instant t. (Gréhaigne, J.F, 1992, 55)
} 


\section{eJRIEPS 23 avril 2011}

3. 2. Les principes tactiques du jeu en déviation

Nous énoncerons ici des principes qui pourront ensuite être réutilisés par les intervenants en Staps. Partant du réel, du « faire », nous y revenons après avoir analysé l'utilisation des techniques de passe dans des contextes d'opposition.

\section{- Une circulation de balle particulière}

L'observation des techniques de déviation utilisées et la quantité d'incertitude produite au cours des séquences offensives montrent que c'est l'enchaînement successif et ininterrompu des différentes directions imposées au ballon qui met la défense en difficulté. Les joueurs créent une circulation de balle systématiquement contradictoire, c'est à dire un enchainement de déviations dont la direction est à chaque fois opposée à la précédente. Ce cheminement imprévisible du ballon ne permet pas au défenseur de modifier sa position, d'engager un déplacement et il le place en « crise de temps » vis-àvis de l'attaquant. Cette circulation du ballon organise le plus souvent un nouveau rapport de forces en faveur de l'attaque par rapport à la défense. Ces résultats confirment une constatation faite par les techniciens quand ils disent qu'il faut «élaborer des passes qui ne soient pas de simples transmissions du ballon, mais par le désarroi qu'elles provoquent au sein des lignes adverses, modifient une situation ou plutôt en créent une autre » (Laurier, 1993, 87).

\section{- Jouer à contre-pied}

Jouer à l'opposé, jouer dans les espaces faibles des défenseurs sont deux principes issus de la pratique que nous confirmons et que nous formaliserons de la façon suivante : pour établir une circulation du ballon ininterrompue et systématiquement contradictoire, les attaquants doivent occuper les espaces libres du jeu avant les défenseurs. Ils doivent utiliser principalement des courses orientées vers la cible en maintenant les distances qui les séparent quasi constantes. Chaque déviation se fera systématiquement dans une direction différente et/ou contradictoire par rapport à la précédente.

\section{- La stabilité spatio-temporelle}

Les attaquants créent et maintiennent un espace de jeu effectif stable dans le temps. C'est la base indispensable à une circulation systématiquement contradictoire du ballon.

\section{- La certitude de la communication}

La technique de la déviation crée une «certitude» dans la communication entre les attaquants. Elle leur permet d'anticiper leurs actions et de prendre de l'avance sur la défense.

\section{- L’auto-organisation}




\section{eJRIEPS 23 avril 2011}

Le jeu en déviation possède les caractéristiques d'un système auto-organisé où les attaquants vont « exploiter le désordre existant pour prendre l'avantage » et exploiter « un meilleur rapport au temps qui permet de gagner grâce à un ordre mis en place plus rapidement que l'adversaire »(Gréhaigne, 2009, 141). La technique de la déviation facilite cette création d'ordre et de prise d'avance des attaquants car l'information transmise entre partenaires est univoque et permet l'anticipation des déplacements et de l'action sur le ballon. Par ailleurs, les postures adoptées par les joueurs, leurs orientations sur le terrain sont des indicateurs pris en compte et organisateurs des déplacements. « La fermeture du couloir de jeu direct par l'adversaire associé à un placement des partenaires dans des espaces laissés libres au sein même de la défense semblent être un signal fort pour déclencher la technique de déviation » (Lemoine \& al, 2007, 89).

\section{Les rapports aux différents registres}

4. 1. Le registre de maitrise

Le jeu en déviation apparaît comme un geste abouti, une excellence technique. Paradoxalement, c'est la technique la plus épurée, la plus simple d'apparence : un seul et unique contact avec la balle qui demande aux joueurs dans un contexte spatio-temporelle contraignant la plus grande habileté.

Nous en déduirons cinq caractéristiques utiles pour l'intervenant en Staps. D'abord la déviation est une «technique de situation » : elle apparait dans des situations de grande densité défensive adverse. Elle est aussi une «technique collective » car le joueur ne peut dévier que s'il a un partenaire disponible. Mais c'est encore une «technique sécurisante », qui éloigne l'adversaire. Une «technique économique » car elle demande peu de courses. Enfin, ce registre de maîtrise s'inscrit dans une «technique du futur », parce qu'il faut jouer avant l'autre.

4. 2. Le registre de lecture

L'utilisation de cette technique par les joueurs s'explique d'abord par une évolution des systèmes de jeu qui privilégient de plus en plus le resserrement défensif. La densité de joueurs au milieu de terrain qui est la zone de transition principale oblige l'équipe en possession du ballon à utiliser des techniques de vitesse de transmission du ballon permettant de dépasser les lignes défensives (Lemoine \& al.., 2007). Adaptée au jeu dans les espaces réduits, la déviation permet de prendre un avantage initial que l'équipe devra conserver jusqu'au tir.

D'autres facteurs comme l'amélioration de la technique individuelle du joueur (vitesse gestuelle) liée aux progrès réalisés dans la fabrication des terrains, du ballon ou encore 


\section{eJRIEPS 23 avril 2011}

des chaussures autorisent une accélération du ballon plus importante que par le passé. Le développement des terrains synthétiques a et aura une influence importante sur la technique du joueur et du jeu. Cette surface permet de gagner en précision dans le jeu car les rebonds intempestifs disparaissent.

\section{4 .3. Le registre de transformation}

Par ailleurs en football comme dans d'autres sports collectifs, l'apprentissage des techniques reste analytique et première. La prise en compte de l'adversaire est seconde. Dans le cas de la technique de déviation, il faut absolument un partenaire pour réceptionner la passe et continuer à jouer. Des propositions didactiques qui incluraient l'adversaire à éliminer par une déviation pourraient être une avancée dans les apprentissages.

\section{Conclusion}

L'approche technologique constitue le moyen d'aborder les faits naturels en les investiguant dans leur complexité en vue de produire des outils de lecture, de compréhension ou de transformation des productions, permettant ainsi d'établir les registres de technicité du domaine abordé. (Bouthier, 2000). Notre travail s'inscrit pleinement dans cette logique et nous avons montré qu'aux différents moments de la construction de l'outil de recueil des données, de l'analyse des résultats ou encore à celui de l'énoncer de principes tactiques, les étapes nécessaires à la construction de compétences professionnelles pour l'enseignant ont été prises en compte à travers cette approche technologique.

Si le joueur acquiert des connaissances et construit des compétences pour agir comme le stipule les programmes de la discipline. L'intervenant, lui, se place à un moment de cette « chaine » de construction de la technique quand il choisit les gestes à construire et la façon dont il souhaite les faire construire par les élèves. L'approche technologique est alors une aide à la décision professionnelle car elle délivre des connaissances qui ont un sens à la fois pratique et théorique pour organiser un enseignement et élaborer des situations d'apprentissage issues de la réalité et qui prennent du sens pour l'apprenant. Dans le cas du jeu en déviation, la technique individuelle replacée dans la dialectique attaque/défense devient une maitrise collective «supérieure » et est une réponse au problème posé par le jeu ce qui est bien la définition à notre sens de la tactique.

Pour l'enseignant qui part du réel pour modifier le réel, la technique de la déviation permet une approche didactique où l'aspect social est fort. Les élèves sont confrontés à la 


\section{eJRIEPS 23 avril 2011}

réalisation d'une «technique collective » où l'autre est indispensable pour construire sa propre technique d'agir sur le ballon.

\section{Bibliographie}

Bouthier, D. (1989). Analyse de la tâche et de l'activité en sports collectifs. In A. Vom Hoffe (Ed), Taches, traitement de l'information et comportement dans les APS (pp. 193-219). Issy les Moulineaux : Ed EAP.

Bouthier D. (2000). L'intervention en EPS, panorama des axes et des types de recherches Conduites ces dernières années. Colloque ARIS, Grenoble 14-16 décembre.

Bouthier, D., \& Durey, A. (1994). Technologie des activités physiques et sportives. Impulsions, 1, 117-126.

Combarnous, M. (1982). La culture face à la technique. Culture technique, 7, 227-241.

Deleplace. R. (1979). Rugby de mouvement-Rugby total. Paris : Edition Revue EPS

Eloi, S. (2009). Style d'un passeur de haut niveau en volley-ball. eJRIEPS, 17, 76-105.

Eloi, S. \& Uhlrich, G. (2001). Contribution à la caractérisation des sports collectifs : les exemples du volley-ball et du rugby. Staps, 56, 109-115.

Falissard, B. (2001). Mesurer la subjectivité en santé, perspective méthodologique et statistique. Paris: Masson.

Grehaigne, J.F. (1992). L'organisation du jeu en football. Joinville : Actio.

Grehaigne, J.F. (2009). Autour du temps. Besançon: Presses Universitaires de Franche Comté.

Gréhaigne, J.F., \& Bouthier, D. (1994). Analyse des évolutions entre deux configurations du jeu en football. Sciences et Motricité, 24, 44-52.

Laurier, A. (1993). Football, culture tactique et principes de jeu (3è éd). Paris: Chiron.

Lemoine, A, Jullien, \& Génolini, C. (2007). Origine et déroulement du jeu en déviation en football. Analyse des mouvements collectifs offensifs au cours de l'Euro 2004. Staps, 77, 79-93.

Martinand, J.L. (1993). Organisation et mise en œuvre des contenus d'enseignement. Recherches en didactiques: contribution à la formation des maitres. Paris : INRP

Parlebas, P. (1967). La sociomotricité. Revue EPS, 86, 7-12

Russo, F. (1986). Introduction à l'histoire des techniques. Paris : Librairie scientifique et technique Albert Blanchard.

Teodoresco, L. (1977). Théorie et méthodologie des jeux sportifs. Paris : Editeurs français réunis. 
eJRIEPS 23 avril 2011

Ulrich, G \& Bouthier, D. (2008). Efficacité d'une situation de formation, par la simulation, à l'observation du mouvement général en rugby. eJRIEPS, 15, 198-215. 